\title{
Aphemia after Infarction of the Left Precentral Gyrus and Premotor Area
}

\author{
Abdullah M. Al-Ajmi, Paul E. Cooper, Rossen T. Rousseff
}

Can J Neurol Sci. 2012; 39: 658-659

The term "aphemia" is now used to describe a motor disorder that affects speech production but leaves the comprehension of spoken and written language intact. Aphemia is a specific form of mutism. Aphemia was formerly referred to as phonetic disintegration, pure anarthria, apraxic dysarthria, cortical dysarthria, verbal apraxia, subcortical motor aphasia, pure motor aphasia, small or mini Broca's aphasia, pure word mutism and kinetic speech production disorder, reflecting the differing views on the nature of the underlying disorder (e.g., aphasia, dysarthria, apraxia). This condition may lie between pure speech disorders (i.e., dysarthrias) and language disorders (i.e., aphasias) and it usually reflects damage in the left frontal operculum ${ }^{1}$. Aphemia may emerge during recovery from more extensive deficits. Occasional cases of progressive aphemia resulting from selective left temporal lobe degeneration have been reported ${ }^{2}$. In addition, cases of epileptic aphemia have been described $^{3}$.

\section{CASE}

A 60-year-old right-handed man presented to the neurology clinic with several complaints. He awoke with an inability to speak, right hand weakness and facial asymmetry. His past history was notable for hypertension. He was taking blood pressure medication.

His general physical examination was unremarkable. During the neurological examination, the patient appeared alert. He had no verbal output and was unable to articulate any sounds, comprehensible or incomprehensible. He could not repeat sounds, hum or sing, but could produce low-pitched guttural moaning. To communicate, he used gestures. The patient had normal comprehension of verbal commands in his native language (Urdu, through an interpreter), Arabic and English. Furthermore he had normal comprehension of the three written languages. His writing was tested in his native language and in English. His penmanship was adversely affected by impaired dexterity; however, his spontaneous writing and writing in response to dictation were accurate. Formal neuropsychological testing was not performed. The patient also had right upper motor neuron-type facial weakness. A slight pronator drift was present on the right side. He had slow and irregular fine finger movements of the right hand and moderately weak finger extension was present in the right hand. Manual strength testing was otherwise normal throughout. Deep tendon reflexes were normal and flexor plantar responses were present. The sensory exam, including responsiveness to light touch, temperature, pinprick and vibration, was normal. The cerebellar examination was normal as were stance and gait.

The patient's presentation was consistent with an ischemic stroke involving the left middle cerebral artery (MCA) distribution. He was admitted to the hospital for a diagnostic work-up and management.

An unenhanced computed tomography scan of the head confirmed an infarction involving the left precentral gyrus and premotor area (Figure).

The blood work showed an elevated low density lipoprotein level of $2.6 \mathrm{mmol} / \mathrm{L}$, but other blood tests were normal. An electrocardiogram showed a normal sinus rhythm and echocardiogram revealed global hypokinesia, a dilated left ventricle and an ejection fraction of $15-20 \%$. The carotid Doppler ultrasound was normal.

The stroke was thought to be cardioembolic in origin and was treated with warfarin (the patient had history of gastric intolerance to aspirin).

After two weeks, the patient regained hand function. He communicated by writing and reported his hand function "back to normal". His facial weakness had also improved, and he was capable of producing incomprehensible sounds when he attempted to say words. Six months later, an interpreter was able to understand the patient's language which sounded "strange/foreign" although both the patient and the interpreter were from the same area. The patient did not show for a further follow up.

\section{Discussion}

The patient was diagnosed as having aphemia. On admission he was mute but had normal comprehension, reading and writing ability. Furthermore, his comprehension of three languages was normal and his written replies to questions were appropriate irrespective of his aphemia when two languages were tested. He had no facial, oral or lingual apraxia.

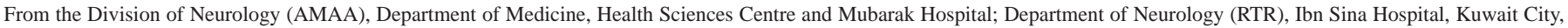
Kuwait; Department of Clinical Neurological Sciences (PEC), University of Western Ontario, London, Ontario, Canada.

Received January 17, 2012. Final Revisions Submitted May 1, 2012.

Correspondence to: Rossen T Rousseff, Department of Neurology, Ibn-Sina Hospital, pob 24527, Safat, 13115, Kuwait City, Kuwait. Email: rossentrousseff@yahoo.co.uk. 


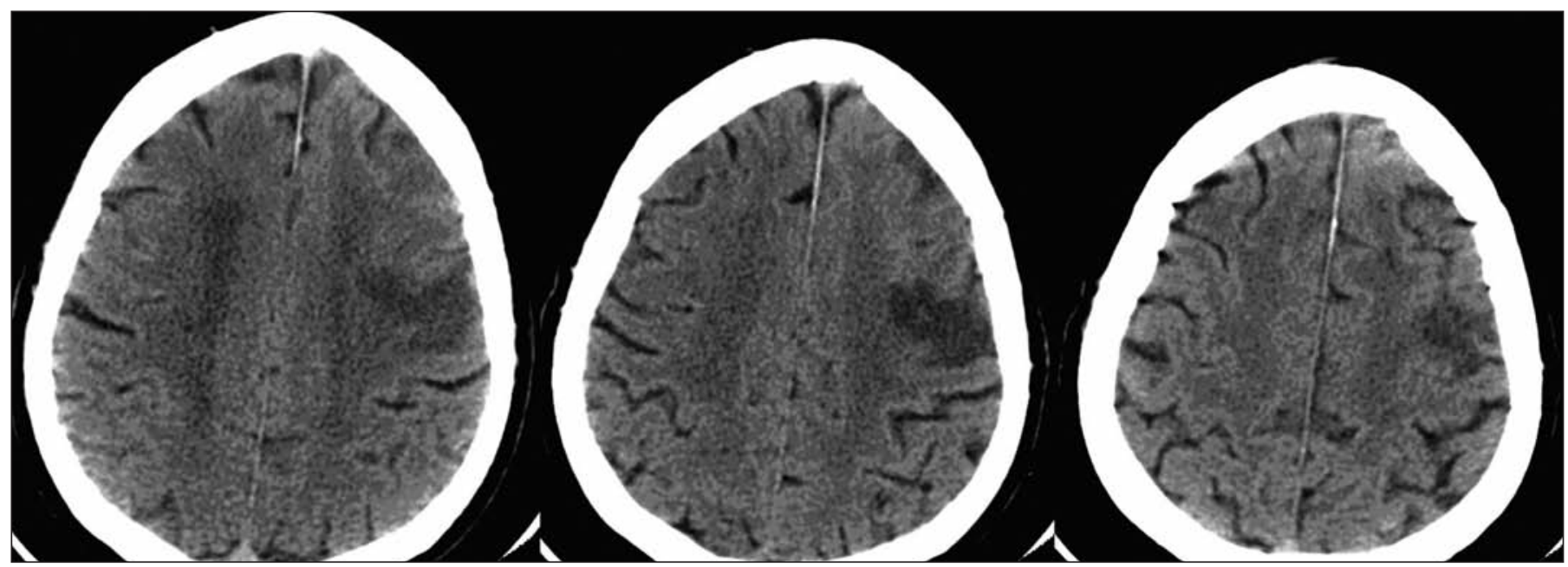

Figure: CT image of infarction involving the left precentral gyrus, extending into the premotor area.

Mutism is the absence of speech output ${ }^{1}$. Several diseases and pathologies at different sites can produce mutism ${ }^{1}$. For example, peripheral disorders, such as laryngitis and vocal cord paralysis, can occasionally cause a mute state. In addition, aphemia, global aphasia, transcortical motor aphasia, and aphasia with subcortical lesions frequently present with mutism. Persistent mutism can be seen with bihemispheric involvement, particularly of the frontal lobes. Furthermore, tissue destruction or dysfunction affecting the upper brain stem or bilaterally affecting the frontal cingulate/supplementary motor area can interfere with the initiation of both behavior and verbal output. When it is severe, this state is called akinetic mutism. Finally, mutism can also be of psychogenic origin.

Broca's aphasia is a nonfluent aphasia with agrammatism, repetition failure, and relatively normal comprehension, usually accompanied by right hemiparesis and oral apraxia. Broca first used the term aphemia (aphemie in French) to designate the syndrome later called "Broca's aphasia". In recent years, however, the term aphemia has been reserved for a syndrome of near-muteness with normal comprehension, reading, and writing. Aphemia is clearly a motor speech disorder rather than an aphasia, because written language and comprehension remain intact. Patients are often anarthric, with no speech whatsoever initially and then effortful, non-fluent speech after sometime. Some patients have persistent dysarthria with dysphonia and sometimes distortions of articulation that sound similar to foreign accents - the "foreign accent syndrome"1 which likely happened to our patient. In aphemia, the communication disturbance involves verbal output and is a speech problem rather than an impairment of language. Aphemia differs from Boca's aphasia because of the absence of agrammatism in spoken and written output; however, controversy remains as to whether aphemia is equivalent to apraxia of speech ${ }^{1,4}$.

Aphemia is associated with anterior lesions of the dominant hemisphere including the pars opercularis, the inferior preRolandic gyrus, or the subjacent white matter ${ }^{4}$. Terao et $\mathrm{al}^{5}$ described three cases in which the patients developed severe dysarthria that temporarily mimicked speech arrest or aphemia.
These cases were due to a localized brain lesion near the left face representation of the human primary motor cortex (face$\mathrm{M} 1)^{5}$. Aphemia can also result from an infarction in the territory of the left MCA, similar to the infarction in our patient, and it often emerges during recovery from more extensive deficits; however, occasional cases of progressive aphemia resulting from selective left temporal lobe degeneration have been reported ${ }^{2}$.

\section{Conclusion}

Multiple disease states and sites of pathology can cause various types of mutism, including aphemia. Aphemia is a rare condition in which patients have impaired speech but intact comprehension and writing. In our patient, aphemia was caused by an infarction in the dominant hemisphere involving the precentral gyrus, extending anteriorly with subjacent white matter involvement.

\section{REFERENCES}

1. Larner AJ. A Dictionary of Neurological Signs. 2nd edition. New York: Springer; 2006. pp. 33-4, 202.

2. Sakurai Y, Murayama S, Fukusako Y, et al. Progressive aphemia in a patient with Pick's disease: a neuropsychological and anatomic study. J Neurol Sci. 1998;159 (2):156-61.

3. Panayiotopoulos CP. Early-onset benign childhood occipital seizure susceptibility syndrome: a syndrome to recognize. Epilepsia. 1999;40(5):621-30.

4. Schiff HB, Alexander MP, Naeser MA, et al. Aphemia: Clinicalanatomic correlations. Arch Neurol. 1983;40:720-7.

5. Terao Y, Ugawa Y, Yamamoto $\mathrm{Y}$ et al. Primary face motor area as the motor representation of articulation. J Neurol. 2007;254(4): $442-7$. 